\title{
Bounds on non-standard interactions of neutrinos from IceCube DeepCore data
}

\author{
S.V. Demidov \\ Institute for Nuclear Research of the Russian Academy of Sciences, \\ 60th October Anniversary prospect 7a, Moscow 117312, Russia \\ E-mail: demidov@ms2.inr.ac.ru
}

\begin{abstract}
New physics in neutrino sector can reveal itself via non-standard neutrino interactions which can result in modification of the standard picture of neutrino propagation in matter. Experiments with atmospheric neutrinos has been used to probe this scenario. Using publicly available three-year low energy data in IceCube DeepCore we place bounds on the parameters $\epsilon_{\alpha \beta}$ of non-standard neutrino interactions in propagation. We obtain restrictive constraints not only for $\mu \tau$ sector but also for flavor changing interactions involving electron neutrinos.
\end{abstract}

Keywords: Beyond Standard Model, Neutrino Physics, Solar and Atmospheric Neutrinos ArXiv EPrint: 1912.04149 


\section{Contents}

1 Introduction 1

2 Description of the analysis 3

$\begin{array}{llc}3 & \text { Results } & 6\end{array}$

$\begin{array}{lll}4 \text { Conclusions } & 10\end{array}$

\section{Introduction}

Phenomenon of neutrino oscillations is well established by many experiments [1]. It implies nontrivial mixing between neutrinos and non-zero values of their masses. Explanation of these properties of neutrinos lies beyond the Standard Model and requires new physics. The latter may reveal itself via non-renormalizable interactions involving neutrino fields. In particular, new Fermi-type interactions of neutrinos with SM fermions are of interest and they are known as non-standard neutrino interactions $[2,3]$. In what follows we consider neutral current (NC) or matter non-standard neutrino interactions (NSI) with the lagrangian

$$
\mathcal{L}_{\mathrm{NSI}}^{\mathrm{NC}}=-\sum_{f, P=P_{L}, P_{R}} \epsilon_{\alpha \beta}^{f P} 2 \sqrt{2} G_{F}\left(\bar{\nu}_{\alpha} \gamma^{\mu} P_{L} \nu_{\beta}\right)\left(\bar{f} \gamma_{\mu} P f\right)
$$

Here $P_{L, R}$ are the chirality projectors, $\epsilon_{\alpha \beta}^{f P}$ are the NSI parameters and sum goes over all SM fermions $f$. Note that the lagrangian (1.1) contains only operators which do not change flavor of the fermion $f$. The flavor changing interactions of the type (1.1) are severely constrained from results on lepton flavor violating and FCNC processes. NC NSI (1.1) can modify neutrino phenomenology in several ways and these interactions can reveal themselves in scattering processes as well as in neutrino oscillation experiments (see refs. [4-7] for reviews). Examples of phenomenologically viable models predicting the matter NSI with sizable values of couplings were discussed e.g. in refs. [8-12].

One of the consequences of the interactions (1.1) is modification of neutrino propagation in matter. In the presence of NSI the evolution of neutrino having energy $E$ is described by the Hamiltonian

$$
H=\frac{1}{2 E} U \operatorname{diag}\left(0, \Delta m_{21}^{2}, \Delta m_{31}^{2}\right) U^{\dagger}+V_{e} \epsilon^{m},
$$

where $U$ is the vacuum Pontecorvo-Maki-Nakagawa-Sakata (PMNS) matrix and $\Delta m_{21}^{2}, \Delta m_{31}^{2}$ are differences of the neutrino masses squared. The last term in (1.2) describes matter effects and it depends on the matter density through $V_{e}=(-) \sqrt{2} G_{F} N_{e}$ for 
(anti)neutrinos, where $N_{e}$ is the electron number density. The NSI parameters from the interaction lagrangian (1.1) enter the Hamiltonian as follows

$$
\epsilon^{m}=\left(\begin{array}{ccc}
1+\epsilon_{e e} & \epsilon_{e \mu} & \epsilon_{e \tau} \\
\epsilon_{e \mu}^{*} & \epsilon_{\mu \mu} & \epsilon_{\mu \tau} \\
\epsilon_{e \tau}^{*} & \epsilon_{\mu \tau}^{*} & \epsilon_{\tau \tau}
\end{array}\right)
$$

where

$$
\epsilon_{\alpha \beta}=\sum_{f=e, u, d}\left(\epsilon_{\alpha \beta}^{f P_{L}}+\epsilon_{\alpha \beta}^{f P_{R}}\right) \frac{N_{f}}{N_{e}} .
$$

Here $N_{f}$ is the number density of the fermion $f$ in matter. In this study we concentrate on neutrino propagation in the Earth and in this case the expression (1.4) for NSI parameters transforms into

$$
\epsilon_{\alpha \beta} \approx \epsilon_{\alpha \beta}^{e V}+3 \frac{N_{u}}{N_{e}} \epsilon_{\alpha \beta}^{u V}+3 \frac{N_{d}}{N_{e}} \epsilon_{\alpha \beta}^{d V}
$$

where $\epsilon_{\alpha \beta}^{f V} \equiv \epsilon_{\alpha \beta}^{f P_{L}}+\epsilon_{\alpha \beta}^{f P_{R}}$. In what follows the notation $\epsilon_{\alpha \beta}$ refers to eq. (1.5). In general the parameters $\epsilon_{\alpha \beta}$ are complex-valued numbers. Here we take them real for simplicity (see [13] for recent discussion of the effect of CP violation in NSI).

Atmospheric neutrinos is an important tool to explore neutrino properties and, in particular, to search for new interactions in neutrino sector. The impact of NSI in propagation at experiments with atmospheric neutrinos has been studied extensively (see [14-25] for an incomplete list). Probes with atmospheric neutrinos has been used to constrain the matter NSI in the Earth. Results of experiments with atmospheric neutrinos allow to put rather stringent bounds [26-30] on the parameters $\epsilon_{\alpha \beta}$. Earlier studies of NSI with IceCube data $[27,29,30]$ put constraints on the NSI parameters in $\mu \tau$ sector. Preliminary results with the bounds on more generic NSI models were recently reported in [31-33] by IceCube collaboration. In the present study we use the publicly available IceCube DeepCore three-year low energy data sample [34] and perform an independent analysis to constrain the parameters $\epsilon_{\alpha \beta}$. This data sample is very close to what was used by IceCube to measure the neutrino oscillation parameters in ref. [35]. We perform an analysis of the NSI effect on atmospheric neutrino propagation in the Earth. Information provided by IceCube with the data release [34] and, in particular, results of Monte-Carlo simulation, expected background from atmospheric muons as well as parametrization of estimated instrumental systematic effects, allows one to made a realistic prediction for number of expected events in models with non-zero matter NSI parameters and compare them against the data. As a result we obtain allowed regions for the parameters $\epsilon_{\alpha \beta}$ under certain model assumptions. We compare them with results from other oscillation experiments and discuss impact of systematic uncertainties.

The rest of the paper is organized as follows. In section 2 we describe methodology used to bound $\epsilon_{\alpha \beta}$ with the IceCube DeepCore three-year data sample. In section 3 we present our results. Section 4 is reserved for conclusions. 


\section{Description of the analysis}

For the present study we use publicly available three-year data sample [34] in IceCube DeepCore which is referred to as 'Sample B' in $^{1}$ ref. [37] and which is very close to what was used in the oscillation analysis [35]. This sample contains 40920 events in total which are distributed over $8 \times 8 \times 2$ binned histogram. The latter is composed of equally spaced bins in $\log _{10} E^{\text {reco }} \in[0.75,1.75], 8$ equally spaced bins in $\cos \theta^{\text {reco }} \in[-1,1]$ as well as 2 bins which correspond to the track-like and cascade-like events. Here $E^{r e c o}$ and $\theta^{\text {reco }}$ refer to reconstructed values of neutrino energy and zenith angle. We use the public IceCube Monte-Carlo provided along with the data sample to model the detector response and to relate physical values of the energy, zenith angle and type of neutrino with reconstructed characteristics of the events. The simulated neutrino sample released at [34] allows to calculate for each bin $i$ the effective areas $A_{i}^{\nu_{\alpha}, t}(E, \cos \theta)$ to be converted with predicted neutrino flux to obtain expected number of events in the bins. The effective areas are obtained as functions of true neutrino energy $E$ in the range from 1 to $1000 \mathrm{GeV}$, zenith angle $\theta$, neutrino type $\nu_{\alpha}$ and type of neutrino interaction $t$ (CC or $\mathrm{NC}$ ).

For prediction of the neutrino flux at the detector level we start with the atmospheric neutrino fluxes $\Phi_{\nu_{\alpha}}^{a t m}(E, \theta)$ for $\nu_{e}, \bar{\nu}_{e}, \nu_{\mu}$ and $\bar{\nu}_{\mu}$ taken from ref. [38]. To describe propagation of the atmospheric neutrinos in the Earth in presence of NSI one should solve the Schrodinger equation with the Hamiltonian (1.2) for the case of varying density. In the present analysis we solve it numerically as described in $[39,40]$ using the algorithm presented in [41]. For calculation of the electron number density in the Earth we use PREM [42]. We fix the following values ${ }^{2}$ of neutrino oscillation parameters: $\sin ^{2} \theta_{12}=0.304, \sin ^{2} \theta_{13}=0.0217$ and $\Delta m_{21}^{2}=7.53 \cdot 10^{-5} \mathrm{eV}^{2}$ and set $\delta_{C P}=0$. Upon obtaining solution to the Schrodinger equation we calculate the transition probabilities $P_{\nu_{\alpha} \rightarrow \nu_{\beta}}(E, \cos \theta)$ to find neutrino of a flavor $\nu_{\beta}$ at the detector level from neutrino of a flavor $\nu_{\alpha}$ produced in the atmosphere. It is the probability functions $P_{\nu_{\alpha} \rightarrow \nu_{\beta}}(E, \cos \theta)$ which depend on the matter NSI parameters $\epsilon_{\alpha \beta}$. The resulting neutrino fluxes at the detector level are then obtained as

$$
\Phi_{\nu_{\beta}}^{\text {det }}(E, \cos \theta)=\sum_{\nu_{\alpha}} \Phi_{\nu_{\alpha}}^{a t m}(E, \cos \theta) P_{\nu_{\alpha} \rightarrow \nu_{\beta}}(E, \cos \theta)
$$

and the expected number of events in $i$-th bin can be found as follows

$$
n_{i}^{\nu}=T \cdot \sum_{t, \nu_{\alpha}} \int d E d \cos \theta A_{i}^{\nu_{\alpha}, t}(E, \cos \theta) \Phi_{\nu_{\alpha}}^{d e t}(E, \cos \theta),
$$

\footnotetext{
${ }^{1}$ Another data sample in this release referred to as 'Sample A' was used for measurement of atmospheric tau neutrino appearance [37] and probing for neutrino mass ordering [36].

${ }^{2}$ We note that analysis of solar neutrino propagation revealed [43] that models with NSI allow for socalled LMA-D solution for the oscillation parameters for which $\sin ^{2} \theta_{12}>0.5$. In this study we do not consider this possibility because, on the one hand, the impact of $\sin ^{2} \theta_{12}$ on the results of our analysis is very mild and, on the other, recent studies (see e.g. [44-46]) showed that the LMA-D scenario is disfavored by experimental data.
} 
where $T$ is the lifetime for the data sample under consideration and sum goes over contributions from different neutrino flavors $\nu_{\alpha}$ (including antineutrinos) and interaction types $t$ (CC or $\mathrm{NC})$.

To compare the expected event distribution with the IceCube DeepCore data we take into account systematic uncertainties and our procedure includes a set of relevant nuisance parameters $\eta_{j}$. They correspond to overall normalization of atmospheric neutrino flux with no prior, the spectral index of the atmospheric neutrino flux with the nominal value $\gamma=$ -2.66 and a prior $\sigma_{\gamma}=0.1$, relative normalizations of $\nu_{e}, \bar{\nu}_{e}$ events and NC events with an uncertainty $20 \%$. Also we include additional corrections to the atmospheric neutrino flux at production to take into account uncertainties in the hadron production in atmosphere. This corrections as functions of neutrino energy and zenith angle have been chosen to reproduce the uncertainties estimated in ref. [47] similar to how it was done in [48]. Also we take into account uncertainty related to the neutrino nucleon cross section with baryon resonance production which is important for low energy part of the neutrino sample. For that we introduce an additional nuisance parameter for the contribution from such type of the events with $40 \%$ uncertainty which is close to what was found in [48]. As discussed in [35] uncertainties on the DIS cross section have negligible impact on the results. We include in the analysis the template for the background of atmospheric muons with corresponding uncorrelated error $\sigma_{\nu, \mu_{a t m}}^{\text {uncor }}$ provided by IceCube for this data sample [34]. Normalization of the background is taken as a nuisance parameter with no prior. Finally, we account for instrumental systematic uncertainties related to the optical efficiencies of DOMs and relevant properties of the ice. These uncertainties are included in our analysis as described in [34]. In summary, our implementation of the systematic uncertainties is very close to how it was done in the original oscillation analysis [35]. We will discuss the impact of the most important systematic errors in the next section.

Let us note, that in general interactions of the type (1.1) result in changes of the NC neutrino-nucleon cross section and thus can modify expected number of events. However, this effect is very model dependent. Not only it depends on other combinations of the parameters $\epsilon_{\alpha \beta}^{f P}$ than those in eq. (1.5), but it is also affected by microscopic model behind the effective lagrangian (1.1) and the resulting cross section will be different fore models with light and heavy mediators In the present analysis we conservatively do not take into account the impact of NSI on the modification on the NC neutrino-nucleon cross sections.

To obtain bounds on the parameters of non-standard neutrino interactions we define (cf. eq. (2) in [35])

$$
\chi^{2}=\sum_{i} \frac{\left(n_{i}^{\nu}+n_{i}^{\mu_{a t m}}-n_{i}^{\text {data }}\right)^{2}}{\left(\sigma_{i}^{\text {data }}\right)^{2}+\left(\sigma_{\nu, \mu_{a t m}, i}^{\text {uncor }}\right)^{2}}+\sum_{j} \frac{\left(\eta_{j}-\hat{\eta}_{j}\right)^{2}}{\sigma_{\eta_{j}}^{2}}
$$

where $n_{i}^{\nu}\left(n_{i}^{\mu_{a t m}}\right)$ is the expected number of events from atmospheric neutrinos (muons), $n_{i}^{\text {data }}$ is the number of data events in the $i$-th bin, $\sigma_{i}^{\text {data }}=\sqrt{n_{i}^{\nu}}$, and the first sum goes over all bins in the data sample. The second term accounts for contribution from the nuisance parameters $\eta_{j}$, given their default values $\hat{\eta}_{j}$ and uncertainties $\sigma_{\eta_{j}}$. We fix $\sin ^{2} \theta_{12}$, $\sin ^{2} \theta_{13}$ and $\Delta m_{21}^{2}$ as described above. We checked that the uncertainties in their values 

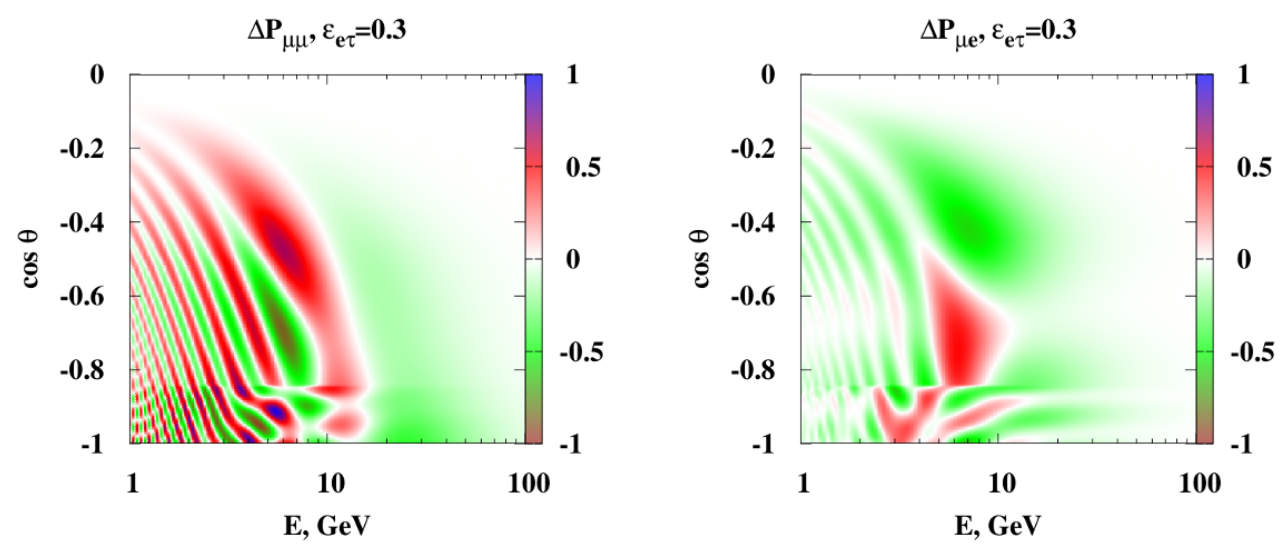

Figure 1. Differences between probabilities $\Delta P_{\mu \mu} \equiv P_{\nu_{\mu} \rightarrow \nu_{\mu}}^{\mathrm{NSI}}-P_{\nu_{\mu} \rightarrow \nu_{\mu}}^{\text {noNSI }}$ (left panel) and $\Delta P_{\mu e} \equiv$ $P_{\nu_{\mu} \rightarrow \nu_{e}}^{\mathrm{NSI}}-P_{\nu_{\mu} \rightarrow \nu_{e}}^{\text {noNSI }}$ (right panel) shown in color as functions of $E$ and $\cos \theta$. The probabilities are calculated with $\epsilon^{e \tau}=0.3$ assuming $\sin ^{2} \theta_{23}=0.51$ and $\Delta m_{31}^{2}=2.5 \times 10^{-3} \mathrm{eV}^{2}$.

produce negligible effect on the final results. Also we assume $\delta_{C P}=0$. Other neutrino oscillation parameters, $\sin ^{2} \theta_{23}$ and $\Delta m_{31}^{2}$, are not fixed to any a priori value but were determined from the analysis itself in most part of the study. As a consistency check we reproduce confidence regions for $\sin ^{2} \theta_{23}$ and $\Delta m_{31}^{2}$ from the IceCube DeepCore data sample assuming $\epsilon_{\alpha \beta}=0$. In particular, we find for the case of normal mass ordering $\sin ^{2} \theta_{23}=0.52_{-0.08}^{+0.07}, \Delta m_{32}^{2}=2.29_{-0.15}^{+0.16} \mathrm{eV}^{2}$ which is very close to those intervals obtained in ref. [35]. In our analysis with non-vanishing matter NSI parameters we consider $\sin ^{2} \theta_{23}$ and $\Delta m_{31}^{2}$ as nuisance parameters with no prior unless stated otherwise. In section 3 we discuss how the bounds on $\epsilon_{\alpha \beta}$ are robust if $\sin ^{2} \theta_{23}$ and $\Delta m_{31}^{2}$ are fixed in the analysis.

The IceCube DeepCore low energy data sample of the atmospheric neutrinos [34] contains (contrary to the earlier IceCube DeepCore oscillation analyses [49]) not only tracklike but also cascade-like events which results mainly from CC interactions of $\nu_{\mu}$ and $\nu_{e}$ (see figure 1 in ref. [35]). This makes the data sample sensitive not only to changes in the muon neutrino flux but also to modification in the flux of electron neutrinos. Most of the theoretical studies discuss impact of the NSI in propagation mainly on the muon neutrino flux $\Phi_{\nu_{\mu}}^{\text {det }}$. This is mostly sufficient for the models with non-zero values of the corresponding parameters in $\mu \tau$ sector where the NSI effect on the electron neutrino flux is very mild given current bounds on the NSI parameters. However, this is not the case for models with non-zero $\epsilon_{e \tau}$ or $\epsilon_{e \mu}$. For illustration, in figures 1 and 2 we show oscillograms for $\Delta P_{\mu \mu}$ and $\Delta P_{\mu e}$ which are the differences between the transition probabilities with and without NSI, i.e. $\Delta P_{\alpha \beta}=P_{\nu_{\alpha} \rightarrow \nu_{\beta}}^{\mathrm{NSI}}-P_{\nu_{\alpha} \rightarrow \nu_{\beta}}^{\mathrm{noNS}}$ for neutrinos. Figure 1 and 2 correspond to $\epsilon_{e \tau}=0.3$ and $\epsilon_{e \mu}=0.3$, respectively. Right panels on both figures correspond to $\Delta P_{\mu \mu}$ while left are reserved for $\Delta P_{\mu e}$. We observe that the off-diagonal matter NSI involving electron neutrinos may considerably modify not only the fluxes of muon neutrinos but also that of electron neutrinos. Note that the effect of non-zero $\epsilon_{e \tau}$ decreases with increase of neutrino energy but this is not the case for $\epsilon_{e \mu}$. Thus we expect that IceCube data for neutrinos at high energies will be also sensitive to the parameter $\epsilon_{e \mu}$. 

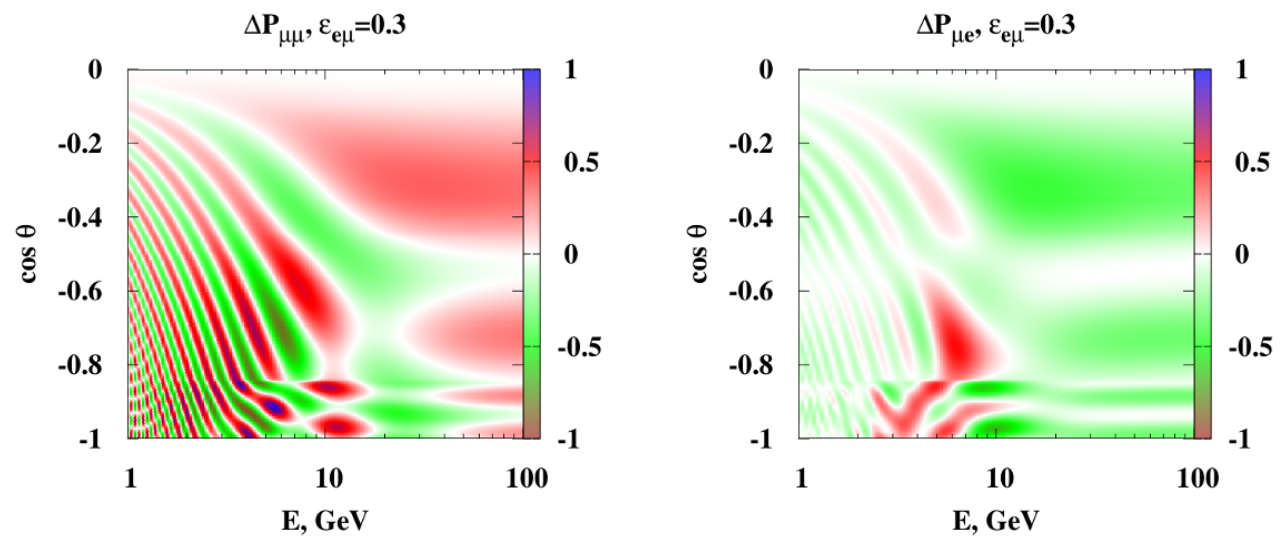

Figure 2. The same as in figure 1 but with $\epsilon_{e \mu}=0.3$.
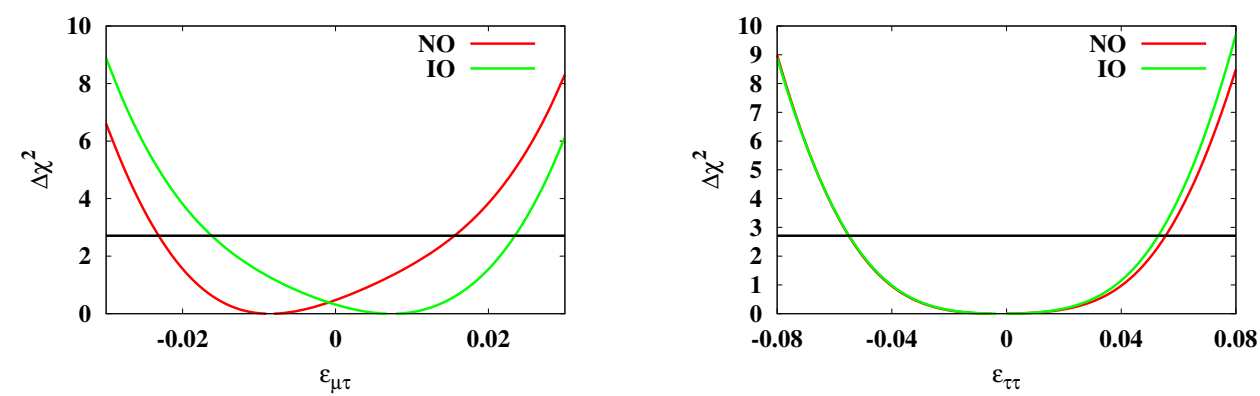

Figure 3. Values of $\Delta \chi^{2}$ for the cases of non-zero $\epsilon_{\mu \tau}$ (left panel) and $\epsilon_{\tau \tau}$ (right panel). Other matter NSI parameters except for $\epsilon_{\mu \tau}$ (left panel) and $\epsilon_{\tau \tau}$ (right panel) are set to zero. Red (dark gray) and green (light gray) lines correspond to normal and inverted neutrino mass hierarchy, respectively.

\section{Results}

In this section we present results on the allowed regions for several matter NSI parameters $\epsilon_{\alpha \beta}$ from the analysis of the low energy three-year IceCube DeepCore data sample [34]. In this study we limit ourselves to a constrained analysis in the matter NSI parameter space assuming only some of $\epsilon_{\alpha \beta}$ to be non-zero. As neutrino oscillation probabilities depend on differences between the diagonal elements $\epsilon_{e e}-\epsilon_{\mu \mu}$ and $\epsilon_{\tau \tau}-\epsilon_{\mu \mu}$ we fix $\epsilon_{\mu \mu}=0$ in what follows.

Let us start with the $\mu \tau$ sector where the NSI parameters, $\epsilon_{\mu \tau}$ and $\epsilon^{\prime} \equiv \epsilon_{\tau \tau}-\epsilon_{\mu \mu}$, are known to be severely constrained from experiments with atmospheric neutrinos $[27,29,30]$. Firstly, we take a single non-zero matter NSI parameter, $\epsilon_{\mu \tau}$ or $\epsilon_{\tau \tau}$, at a time and perform minimization of $\chi^{2}$ given by eq. (2.3) with respect to all other variables (including $\Delta m_{31}^{2}$, $\sin ^{2} \theta_{23}$ and other nuisance parameters discussed in previous section). We consider the cases of normal (NO) and inverted (IO) neutrino mass ordering independently. The results for $\Delta \chi^{2} \equiv \chi^{2}-\chi_{\min }^{2}$, where $\chi_{\min }^{2}$ is an absolute minimum of $\chi^{2}$ for each case, are shown in figure 3 for non-zero $\epsilon_{\mu \tau}$ (left panel) and $\epsilon_{\tau \tau}$ (right panel). We find the following single 

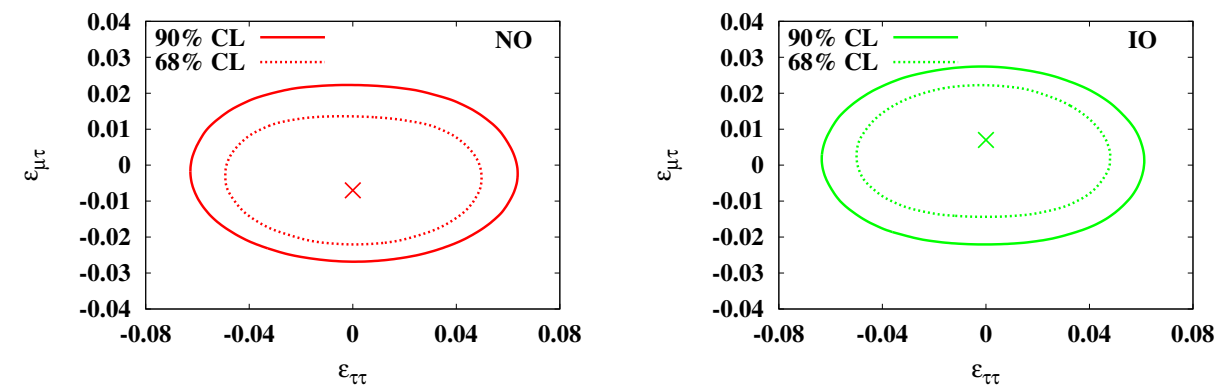

Figure 4. Allowed regions for the parameters $\epsilon_{\mu \tau}$ and $\epsilon_{\tau \tau}$ for NO (left panel) and IO (right panel). Other parameters $\epsilon_{\alpha \beta}$ of the matter NSI are set to zero.

parameter allowed ranges at $90 \%$ C.L.

$$
\begin{aligned}
& -0.023<\epsilon_{\mu \tau}<0.016(\mathrm{NO}), \quad-0.016<\epsilon_{\mu \tau}<0.023(\mathrm{IO}), \\
& -0.055<\epsilon_{\tau \tau}<0.056(\mathrm{NO}), \quad-0.055<\epsilon_{\tau \tau}<0.053(\mathrm{IO}) .
\end{aligned}
$$

Note that the obtained regions for $\epsilon_{\mu \tau}$ are consistent ${ }^{3}$ with those $-0.020<\epsilon_{\mu \tau}<0.024$ (NO) obtained by IceCube collaboration [30] in a similar single parameter analysis using three years of their data with upward going track events selection. The regions (3.1), (3.2) are also close to the preliminary IceCube bounds $\left|\epsilon_{\mu \tau}\right| \lesssim 0.17$ and $\left|\epsilon_{\tau \tau}\right| \lesssim 0.04$ (NO) from an analysis of DeepCore data reported ${ }^{4}$ in [33].

Next, we consider the NSI models in which both parameters in the $\mu \tau$ sector, i.e. $\epsilon_{\mu \tau}$ and $\epsilon_{\tau \tau}$. are not equal to zero. Corresponding allowed regions on $\left(\epsilon_{\mu \tau}, \epsilon_{\tau \tau}\right)$ plane are presented in figure 4 for NO (left panel) and IO (right panel). After marginalization with respect to each of these parameters we obtain the following allowed ranges at $90 \%$ C.L.

$$
\begin{array}{ll}
-0.027<\epsilon_{\mu \tau}<0.022(\mathrm{NO}), & -0.022<\epsilon_{\mu \tau}<0.027(\mathrm{IO}), \\
-0.063<\epsilon_{\tau \tau}<0.064(\mathrm{NO}), & -0.064<\epsilon_{\tau \tau}<0.061(\mathrm{IO}),
\end{array}
$$

which are only slightly wider than those found in the single parameter analysis and shown in (3.1), (3.2). The marginalized bounds on $\epsilon_{\mu \tau}$ are weaker than the allowed range $-0.018<$ $\epsilon_{\mu \tau}<0.016$ (NO), 90\% C.I. obtained in ref. [29] from the analysis of a one year high energy IceCube data sample [50] and than the earlier bound $-0.018<\epsilon_{\mu \tau}<0.017$ (NO) at $90 \%$ C.L. obtained in ref. [27] using the data from 79-string IceCube configuration and DeepCore. At the same time the marginalized bounds (3.4) on the parameter $\epsilon^{\prime} \equiv \epsilon_{\tau \tau}$ are somewhat better than the allowed range $-0.11<\epsilon^{\prime}<0.09$ at $90 \%$ C.L. from ref. [27] and the constraint $\left|\epsilon^{\prime}\right|<0.15$ obtained by Super-Kamiokande experiment [26]. Comparable bound on $\epsilon^{\prime}$ was obtained in ref. [29] from a combination of high energy IceCube data and Super-Kamiokande results. Let us note that the effect of $\epsilon^{\prime}$ on neutrino propagation

\footnotetext{
${ }^{3}$ With our convention (1.4) the values of $\epsilon_{\alpha \beta}$ differ by a factor of $r \equiv N_{d} / N_{e} \approx 3$ from those used e.g. in $[26,29,30]$.

${ }^{4}$ Note that the analysis [33] assumes presence of the complex phases in the flavour changing NSI parameters. For consistency, here we cite preliminary bounds for real valued parameters.
} 

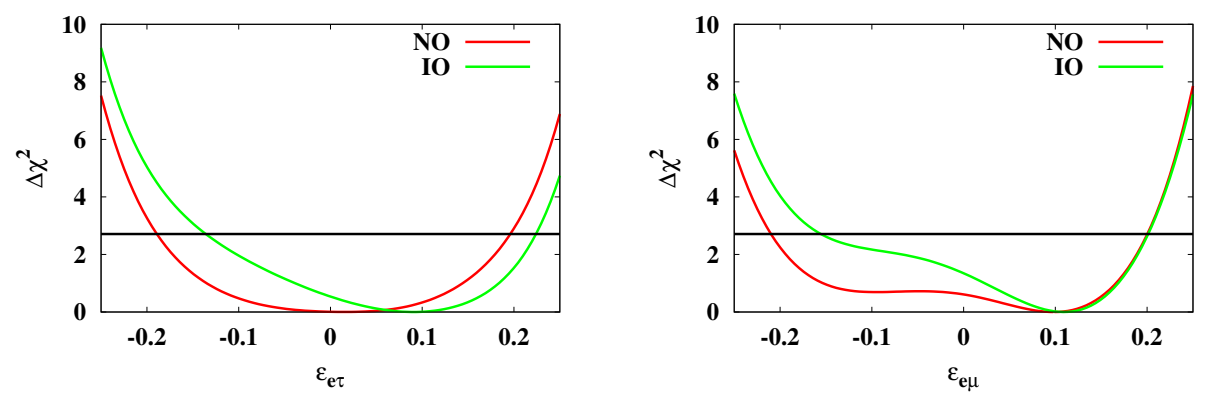

Figure 5. Values of $\Delta \chi^{2}$ for the cases of non-zero $\epsilon_{e \tau}$ (left panel) and $\epsilon_{e \mu}$ (right panel). Other NSI parameters except for $\epsilon_{e \tau}$ (left panel) and $\epsilon_{e \mu}$ (right panel) are set to zero. Red (dark gray) and green (light gray) lines correspond to NO and IO, respectively.
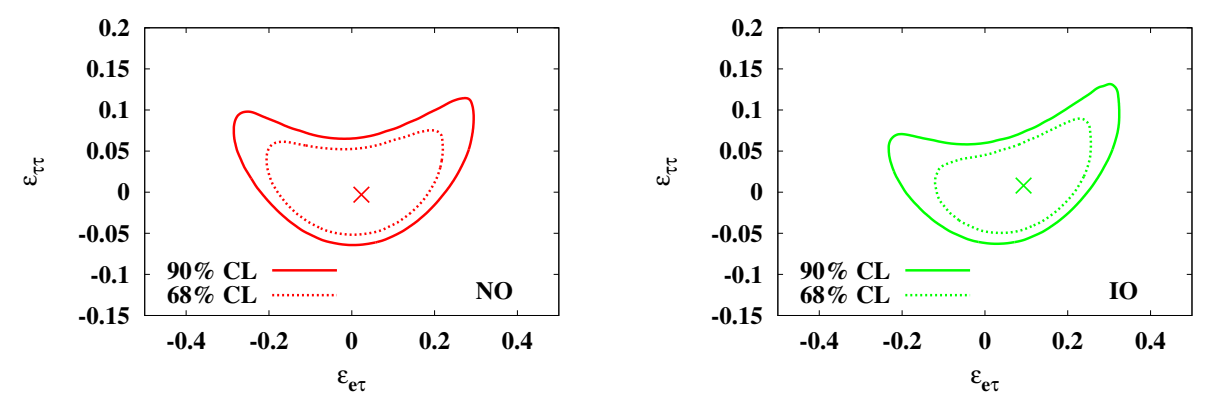

Figure 6. Allowed regions for the parameters $\epsilon_{e \tau}$ and $\epsilon_{\tau \tau}$ for NO (left panel) and IO (right panel). Other parameters of the matter NSI including $\epsilon_{e e}$ are set to zero.

decreases with neutrino energy (see e.g. [27]) and thus to probe this parameter one should rely on low energy part of the atmospheric neutrino spectrum.

Now let us turn to the NSI models with non-zero parameters involving electron neutrinos. In figure 5 we show $\Delta \chi^{2}$ for NSI models with non-zero flavor changing parameters $\epsilon_{e \tau}$ (left panel) and $\epsilon_{e \mu}$ (right panel) taking a single non-zero NSI parameter at a time. Corresponding allowed regions at $90 \%$ C.L. read

$$
\begin{aligned}
& -0.21<\epsilon_{e \mu}<0.20(\mathrm{NO}), \quad-0.16<\epsilon_{e \mu}<0.20(\mathrm{IO}), \\
& -0.19<\epsilon_{e \tau}<0.20(\mathrm{NO}), \quad-0.14<\epsilon_{e \tau}<0.22(\mathrm{IO}) .
\end{aligned}
$$

The allowed single parameter bounds for $\epsilon_{e \mu}$ and $\epsilon_{e \tau}$ are consistent with the preliminary results $\left|\epsilon_{e \mu}\right| \lesssim 0.16$ and $\left|\epsilon_{e \tau}\right| \lesssim 0.2$ (NO) reported by IceCube [33]. Note that based on the behaviour of $\Delta P_{\mu \mu}$ and $\Delta P_{\mu e}$ shown in figure 2 one can expect an improvement in the allowed ranges on $\epsilon_{e \mu}$ with data on atmospheric neutrino at high energies. It is well known that the effect of $\epsilon_{e \tau}$ on neutrino transition probabilities strongly depends on the values of other matter NSI parameters. Following the analysis [26] and earlier studies $[15,17]$ let us consider the NSI models with non-zero parameters in $e \tau$ sector, i.e. $\epsilon_{e e}, \epsilon_{e \tau}$ and $\epsilon_{\tau \tau}$. In figure 6 we show allowed regions for $\epsilon_{e \tau}$ and $\epsilon_{\tau \tau}$ within these models assuming $\epsilon_{e e}=0$. The parabolic form of the allowed region comes from the approximate relation $\epsilon_{\tau \tau} \sim$ $\frac{\left|\epsilon_{e \tau}\right|^{2}}{1+\epsilon_{e e}}$, which should be satisfied for consistency with high energy part of the atmospheric 

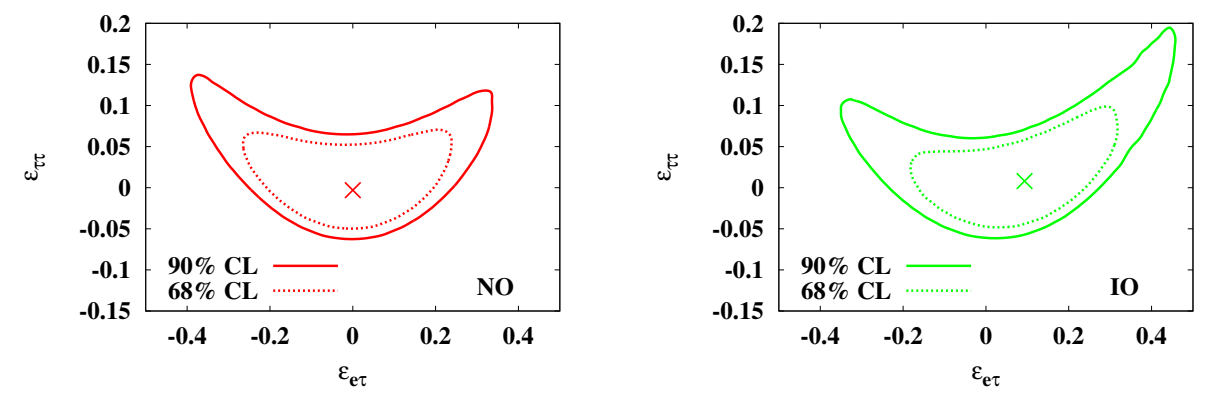

Figure 7. The same as in figure 6 but for $\epsilon_{e e}=0.2$.
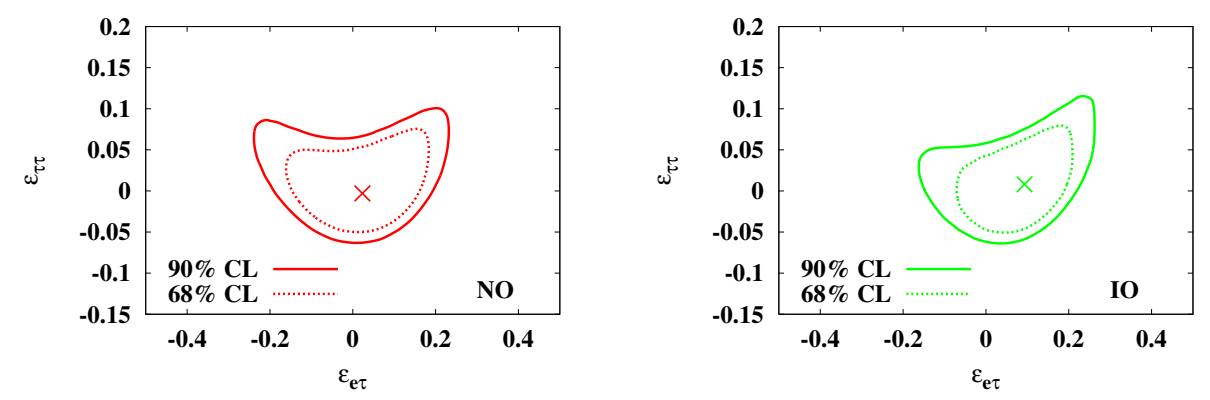

Figure 8. The same as in figure 6 but for $\epsilon_{e e}=-0.2$.

neutrino spectrum, see [15, 17]. Similar allowed regions from preliminary analysis of treeyear DeepCore data were presented in [31]. The sensitivity of the considered dataset to $\epsilon_{e e}$ is relatively weak, but still its non-zero value may affect the bounds on $\epsilon_{e \tau}$ and $\epsilon_{\tau \tau}$ in this class of NSI models through the above relation. As an illustration, in figures 7 and 8 are shown the allowed regions in $\left(\epsilon_{\tau \tau}, \epsilon_{e \tau}\right)$ plane assuming $\epsilon_{e e}=0.2$ and -0.2 , respectively. We see the dependence of the bounds for $\epsilon_{e \tau}$ on the assumption about the value of $\epsilon_{e e}$. The same is valid for allowed regions for $\epsilon_{\tau \tau}$ which can be considerably modified as compared to those given by eq. (3.4) obtained for the NSI models with other $\epsilon_{\alpha \beta}=0$. The found allowed regions for $\epsilon_{e \tau}$ and $\epsilon_{\tau \tau}$ are compatible to the latest constraints on these parameters obtained with the Super-Kamiokande data in ref. [28] using somewhat different anzatz for the parameter space.

Finally we study impact of different nuisance parameters discussed in section 2 on our results. Firstly, in figure 9 we present $\Delta \chi^{2}$ obtained in the single-parameter analyses (for NSI models with non-zero $\epsilon_{\mu \tau}, \epsilon_{\tau \tau}, \epsilon_{e \tau}$ or $\epsilon_{e \mu}$ and assuming normal neutrino mass ordering) for the case with all systematic uncertainties included (which is our default analysis) in comparison with those obtained with (a) fixed nuisance parameters related directly to the uncertainties in atmospheric neutrino flux, i.e. spectral index and uncertainties in the hadron production in atmosphere, (b) fixed nuisance parameters related to the experimental systematic uncertainties and (c) fixed nuisance parameters which determine normalizations of different contributions to expected number of events including those related to the neutrino nucleon cross section and atmospheric muon background. For illustration we present results for the case of normal mass ordering. One observes that the 

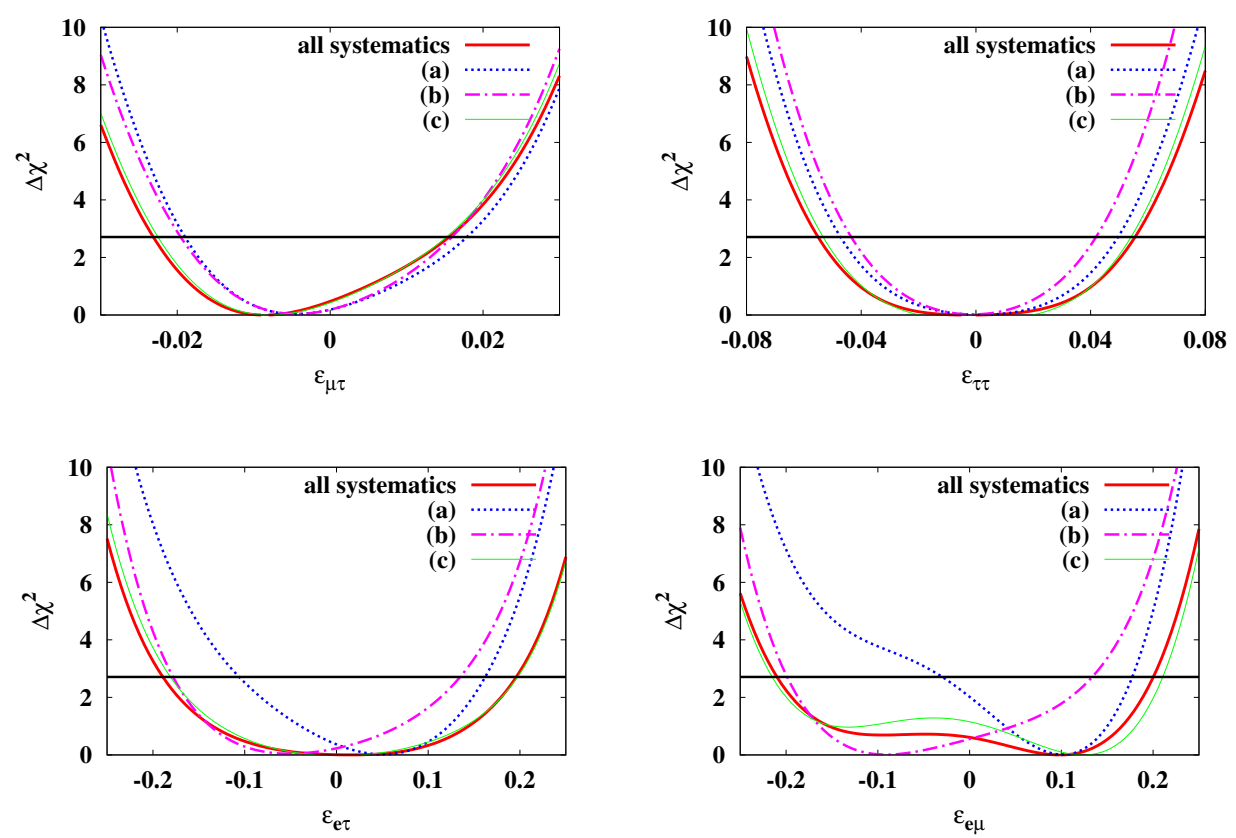

Figure 9. Values of $\Delta \chi^{2}$ (NO) for the NSI models with non-zero values of $\epsilon_{\mu \tau}$ (upper left), $\epsilon_{\tau \tau}$ (upper right), $\epsilon_{e \tau}$ (lower left) and $\epsilon_{e \mu}$ (lower right) obtained in the analyses with all systematic uncertainties included (red thick solid line) in comparison with those obtained with (a) fixed nuisance parameters related to atmospheric neutrino flux (blue dotted line), (b) fixed nuisance parameters related to the experimental uncertainties (magenta dashed-dotted line) and (c) fixed nuisance parameters related to normalization of different contributions to expected number of events (green thin solid line).

most important systematic uncertainties are the experimental ones and those related to atmospheric neutrino flux, specifically for the NSI models with non-zero $\epsilon_{e \tau}$ and $\epsilon_{\mu \tau}$. We also study impact of neutrino oscillation parameters, i.e. $\sin ^{2} \theta_{23}$ and $\Delta m_{31}^{2}$. As an example, we fix them to their optimal values without NSI for the case of normal mass ordering (see section 2). Corresponding allowed ranges for $\epsilon_{\mu \tau}, \epsilon_{\tau \tau}, \epsilon_{e \tau}, \epsilon_{e \mu}$ (a single non-zero NSI parameter at a time) becomes

$$
\begin{array}{lr}
-0.19<\epsilon_{\mu \tau}<0.13, & -0.052<\epsilon_{\tau \tau}<0.051 \\
-0.17<\epsilon_{e \tau}<0.16, & -0.20<\epsilon_{e \mu}<0.18
\end{array}
$$

By comparison with (3.1), (3.2) and (3.5), (3.6) we see that the oscillation parameters affect very little these bounds.

\section{Conclusions}

Let us summarize results of our study. Here we used the three-year IceCube DeepCore data sample [34] of low energy atmospheric neutrinos to constrain the parameters of nonstandard neutrino interactions in propagation. This data sample contains track-like as well as cascade-like events which makes it sensitive not mainly to muon but also to electron 
neutrino flux at the detector level. Using this dataset we found the bounds on several $\epsilon_{\alpha \beta}$ under different model assumptions. In particular, we presented allowed regions for the matter NSI parameters, assuming 1) single non-zero parameter at a time, 2) non-zero parameters in $\mu \tau$ sector and 3) non-zero $\epsilon_{e \tau}$ and $\epsilon_{\tau \tau}$ for several fixed values of $\epsilon_{e e}$. The bounds on $\epsilon_{\mu \tau}$ and $\epsilon_{\tau \tau}$ were found to be close to those [27-30] extracted from different data samples of the IceCube/DeepCore as well as from Super-Kamiokande results. Obtained single-parameter bounds on $\epsilon_{\mu \tau}, \epsilon_{\tau \tau}, \epsilon_{e \tau}$ and $\epsilon_{e \mu}$ are consistent with the preliminary IceCube results [31-33] from a similar analysis of three-year DeepCore data. We studied the impact of different sources of systematic uncertainties and found that the obtained bounds are mainly stable with respect to the nuisance parameters of the analysis. The main errors in the bounds results from uncertainties in the atmospheric neutrino flux and from experimental uncertainties. They are specifically important for single parameter bounds on $\epsilon_{e \tau}$ and $\epsilon_{e \mu}$. Note that from energy dependence of neutrino transition probabilities we can expect an improvement in the bound on $\epsilon_{e \mu}$ using studies with high energy part of atmospheric neutrino spectrum.

In the main text we made a comparison mainly with the results obtained from oscillation experiments with atmospheric neutrinos. The obtained bounds on $\epsilon_{\mu \tau}, \epsilon_{\tau \tau}, \epsilon_{e \tau}$ and $\epsilon_{e \mu}$ are also consistent with the results of global analysis of neutrino oscillation experiments [51] (see also $[22,52,53]$ for earlier studies) obtained under assumptions that the matter NSI are given by neutrino interactions with quarks only and that their flavour structure is independent of the quark flavour. Further insight on possible strength of NSI can be obtained by combining with the results of scattering experiments [46, 54] (see also [55, 56]). Here we were interested in the impact of the low energy IceCube DeepCore data [34] solely and leaved a combined analysis for future study.

\section{Acknowledgments}

I am grateful to Philipp Eller for helpful correspondence. The work was supported by the RSF grant 17-12-01547.

Open Access. This article is distributed under the terms of the Creative Commons Attribution License (CC-BY 4.0), which permits any use, distribution and reproduction in any medium, provided the original author(s) and source are credited.

\section{References}

[1] Particle Data Group collaboration, Review of particle physics, Phys. Rev. D 98 (2018) 030001 [INSPIRE].

[2] L. Wolfenstein, Neutrino oscillations in matter, Phys. Rev. D 17 (1978) 2369 [INSPIRE].

[3] M.M. Guzzo, A. Masiero and S.T. Petcov, On the MSW effect with massless neutrinos and no mixing in the vacuum, Phys. Lett. B 260 (1991) 154 [INSPIRE].

[4] T. Ohlsson, Status of non-standard neutrino interactions, Rept. Prog. Phys. 76 (2013) 044201 [arXiv: 1209.2710] [INSPIRE]. 
[5] O.G. Miranda and H. Nunokawa, Non standard neutrino interactions: current status and future prospects, New J. Phys. 17 (2015) 095002 [arXiv: 1505. 06254] [InSPIRE].

[6] Y. Farzan and M. Tortola, Neutrino oscillations and non-standard interactions, Front. in Phys. 6 (2018) 10 [arXiv:1710.09360] [INSPIRE].

[7] P.S. Bhupal Dev et al., Neutrino non-standard interactions: a status report, SciPost Phys. Proc. 2 (2019) 001 [arXiv: 1907.00991] [InSPIRE].

[8] Y. Farzan, A model for large non-standard interactions of neutrinos leading to the LMA-Dark solution, Phys. Lett. B 748 (2015) 311 [arXiv:1505.06906] [INSPIRE].

[9] Y. Farzan and I.M. Shoemaker, Lepton flavor violating non-standard interactions via light mediators, JHEP 07 (2016) 033 [arXiv:1512.09147] [INSPIRE].

[10] Y. Farzan and J. Heeck, Neutrinophilic nonstandard interactions, Phys. Rev. D 94 (2016) 053010 [arXiv: 1607.07616] [INSPIRE].

[11] Y. Farzan, Viable models for large non-standard neutrino interactions, arXiv:1612.04971 [INSPIRE].

[12] K.S. Babu, P.S.B. Dev, S. Jana and A. Thapa, Non-standard interactions in radiative neutrino mass models, JHEP 03 (2020) 006 [arXiv:1907.09498] [INSPIRE].

[13] I. Esteban, M.C. Gonzalez-Garcia and M. Maltoni, On the determination of leptonic $C P$-violation and neutrino mass ordering in presence of non-standard interactions: present status, JHEP 06 (2019) 055 [arXiv:1905.05203] [INSPIRE].

[14] M.C. Gonzalez-Garcia and M. Maltoni, Atmospheric neutrino oscillations and new physics, Phys. Rev. D 70 (2004) 033010 [hep-ph/0404085] [INSPIRE].

[15] A. Friedland, C. Lunardini and M. Maltoni, Atmospheric neutrinos as probes of neutrino-matter interactions, Phys. Rev. D 70 (2004) 111301 [hep-ph/0408264] [InSPIRE].

[16] M. Blennow, T. Ohlsson and W. Winter, Non-standard Hamiltonian effects on neutrino oscillations, Eur. Phys. J. C 49 (2007) 1023 [hep-ph/0508175] [INSPIRE].

[17] A. Friedland and C. Lunardini, A test of $\tau$ neutrino interactions with atmospheric neutrinos and K2K, Phys. Rev. D 72 (2005) 053009 [hep-ph/0506143] [INSPIRE].

[18] M.C. Gonzalez-Garcia, F. Halzen and M. Maltoni, Physics reach of high-energy and high-statistics IceCube atmospheric neutrino data, Phys. Rev. D 71 (2005) 093010 [hep-ph/0502223] [INSPIRE].

[19] W.A. Mann, D. Cherdack, W. Musial and T. Kafka, Apparent multiple $\Delta m_{32}^{2}$ in muon anti-neutrino and muon neutrino survival oscillations from non-standard interaction matter effect, Phys. Rev. D 82 (2010) 113010 [arXiv: 1006.5720] [InSPIRE].

[20] T. Ohlsson, H. Zhang and S. Zhou, Effects of nonstandard neutrino interactions at PINGU, Phys. Rev. D 88 (2013) 013001 [arXiv:1303.6130] [InSPIRE].

[21] S. Choubey and T. Ohlsson, Bounds on non-standard neutrino interactions using PINGU, Phys. Lett. B 739 (2014) 357 [arXiv:1410.0410] [INSPIRE].

[22] M.C. Gonzalez-Garcia, M. Maltoni and J. Salvado, Testing matter effects in propagation of atmospheric and long-baseline neutrinos, JHEP 05 (2011) 075 [arXiv:1103.4365] [INSPIRE].

[23] I. Mocioiu and W. Wright, Non-standard neutrino interactions in the mu-tau sector, Nucl. Phys. B 893 (2015) 376 [arXiv: 1410.6193] [INSPIRE]. 
[24] A. Chatterjee, P. Mehta, D. Choudhury and R. Gandhi, Testing nonstandard neutrino matter interactions in atmospheric neutrino propagation, Phys. Rev. D 93 (2016) 093017 [arXiv: 1409.8472] [INSPIRE].

[25] S. Fukasawa and O. Yasuda, The possibility to observe the non-standard interaction by the Hyperkamiokande atmospheric neutrino experiment, Nucl. Phys. B 914 (2017) 99 [arXiv: 1608.05897] [INSPIRE].

[26] Super-Kamiokande collaboration, Study of non-standard neutrino interactions with atmospheric neutrino data in Super-Kamiokande I and II, Phys. Rev. D 84 (2011) 113008 [arXiv: 1109.1889] [INSPIRE].

[27] A. Esmaili and A.Yu. Smirnov, Probing non-standard interaction of neutrinos with IceCube and DeepCore, JHEP 06 (2013) 026 [arXiv: 1304.1042] [INSPIRE].

[28] S. Fukasawa and O. Yasuda, Constraints on the nonstandard interaction in propagation from atmospheric neutrinos, Adv. High Energy Phys. 2015 (2015) 820941 [arXiv:1503.08056] [INSPIRE].

[29] J. Salvado, O. Mena, S. Palomares-Ruiz and N. Rius, Non-standard interactions with high-energy atmospheric neutrinos at IceCube, JHEP 01 (2017) 141 [arXiv:1609.03450] [INSPIRE].

[30] ICECuBE collaboration, Search for nonstandard neutrino interactions with IceCube DeepCore, Phys. Rev. D 97 (2018) 072009 [arXiv:1709.07079] [InSPIRE].

[31] T. Ehrhardt, Search for NSI with IceCube DeepCore, presented at the Advanced Workshop on Physics of Atmospheric Neutrinos (PANE 2018), May 28-June 1, Trieste, Italy (2018).

[32] C. de los Heros, Particle physics with IceCube, talk given at the XVIII International Workshop on Neutrino Telescopes, March 18-22, Venice, Italy (2019).

[33] T. Ehrhardt, Search for NSI in neutrino propagation with IceCube DeepCore, talk given at the $4^{\text {th }}$ Uppsala workshop on Particle Physics with Neutrino Telescopes (PPNT19), October 7-9, Uppsala, Sweden (2019).

[34] ICECuBE collaboration, Three-year high-statistics neutrino oscillation samples, https://icecube.wisc.edu/science/data/highstats_nuosc_3y.

[35] ICECUBE collaboration, Measurement of atmospheric neutrino oscillations at 6-56 GeV with IceCube DeepCore, Phys. Rev. Lett. 120 (2018) 071801 [arXiv:1707.07081] [INSPIRE].

[36] ICECuBE collaboration, Development of an analysis to probe the neutrino mass ordering with atmospheric neutrinos using three years of IceCube DeepCore data, Eur. Phys. J. C 80 (2020) 9 [arXiv: 1902.07771] [INSPIRE].

[37] ICECuBE collaboration, Measurement of atmospheric tau neutrino appearance with IceCube DeepCore, Phys. Rev. D 99 (2019) 032007 [arXiv:1901.05366] [InSPIRE].

[38] M. Honda et al., Atmospheric neutrino flux calculation using the NRLMSISE-00 atmospheric model, Phys. Rev. D 92 (2015) 023004 [arXiv: 1502.03916] [InSPIRE].

[39] M.M. Boliev et al., Search for muon signal from dark matter annihilations inthe Sun with the Baksan Underground Scintillator Telescope for 24.12 years, JCAP 09 (2013) 019 [arXiv: 1301.1138] [INSPIRE].

[40] S.V. Demidov, Non-standard interactions and neutrinos from dark matter annihilation in the Sun, JCAP 02 (2018) 001 [arXiv:1711.00911] [INSPIRE]. 
[41] T. Ohlsson and H. Snellman, Three flavor neutrino oscillations in matter, J. Math. Phys. 41 (2000) 2768 [Erratum ibid. 42 (2001) 2345] [hep-ph/9910546] [INSPIRE].

[42] A.M. Dziewonski and D.L. Anderson, Preliminary reference Earth model, Phys. Earth Planet. Inter. 25 (1981) 297.

[43] O.G. Miranda, M.A. Tortola and J.W.F. Valle, Are solar neutrino oscillations robust?, JHEP 10 (2006) 008 [hep-ph/0406280] [INSPIRE].

[44] P. Coloma, M.C. Gonzalez-Garcia, M. Maltoni and T. Schwetz, COHERENT enlightenment of the neutrino dark side, Phys. Rev. D 96 (2017) 115007 [arXiv:1708.02899] [INSPIRE].

[45] C. Giunti, General COHERENT constraints on neutrino nonstandard interactions, Phys. Rev. D 101 (2020) 035039 [arXiv:1909.00466] [INSPIRE].

[46] P. Coloma, I. Esteban, M.C. Gonzalez-Garcia and M. Maltoni, Improved global fit to Non-Standard neutrino Interactions using COHERENT energy and timing data, JHEP 02 (2020) 023 [arXiv : 1911.09109] [INSPIRE].

[47] G.D. Barr, T.K. Gaisser, S. Robbins and T. Stanev, Uncertainties in atmospheric neutrino fluxes, Phys. Rev. D 74 (2006) 094009 [astro-ph/0611266] [INSPIRE].

[48] A. Terliuk, Measurement of atmospheric neutrino oscillations and search for sterile neutrino mixing with IceCube DeepCore, Ph.D. thesis, Humboldt University, Berlin, Germany (2018).

[49] ICECuBE collaboration, Determining neutrino oscillation parameters from atmospheric muon neutrino disappearance with three years of IceCube DeepCore data, Phys. Rev. D 91 (2015) 072004 [arXiv: 1410.7227] [INSPIRE].

[50] IceCube collaboration, Searches for sterile neutrinos with the IceCube detector, Phys. Rev. Lett. 117 (2016) 071801 [arXiv: 1605.01990] [INSPIRE].

[51] I. Esteban et al., Updated constraints on non-standard interactions from global analysis of oscillation data, JHEP 08 (2018) 180 [arXiv:1805.04530] [INSPIRE].

[52] M.C. Gonzalez-Garcia and M. Maltoni, Determination of matter potential from global analysis of neutrino oscillation data, JHEP 09 (2013) 152 [arXiv:1307.3092] [INSPIRE].

[53] M.C. Gonzalez-Garcia, M. Maltoni and T. Schwetz, Global analyses of neutrino oscillation experiments, Nucl. Phys. B 908 (2016) 199 [arXiv: 1512.06856] [INSPIRE].

[54] P. Coloma et al., Curtailing the dark side in non-standard neutrino interactions, JHEP 04 (2017) 116 [arXiv: 1701.04828] [INSPIRE].

[55] J. Barranco, O.G. Miranda, C.A. Moura and J.W.F. Valle, Constraining non-standard neutrino-electron interactions, Phys. Rev. D 77 (2008) 093014 [arXiv:0711.0698] [InSPIRE].

[56] C. Biggio, M. Blennow and E. Fernandez-Martinez, General bounds on non-standard neutrino interactions, JHEP 08 (2009) 090 [arXiv: 0907.0097] [INSPIRE]. 